\title{
Population dynamics in a metastable neon magneto-optical trap
}

\author{
R. D. Glover ${ }^{1}$, J. Calvert ${ }^{1,2}$, and R. T. Sang ${ }^{1,2}$ \\ ${ }^{1}$ Centre for Quantum Dynamics, Griffith University, Nathan QLD 4111, Australia and \\ ${ }^{2}$ ARC Centre of Excellence for Coherent X-Ray Science, Griffith University, Nathan, Australia $4111^{*}$
}

(Dated: January 25, 2013)

\begin{abstract}
We observe the population dynamics within a metastable neon magneto-optical trap (MOT) through the measurement of the average squared Clebsch-Gordan coefficient, $C^{2}$, over a range of laser detunings. The magnitude of $C^{2}$ is dependent on the internal quantum state of an atom interacting with the light field and is found to show a strong dependence on the applied laser detuning. Previously it has been reported [C. G. Townsend et al, Phys. Rev. A 52, 1423 (1995)] that trapped atoms in a MOT are pumped towards states that interact the most strongly with the local field and therefore the measured value of $C^{2}$ is larger than the average over all possible transitions. For the ${ }^{3} \mathrm{P}_{2}$ to ${ }^{3} \mathrm{D}_{3}$ cooling transition in metastable neon the average $C^{2}$ value is equal to 0.46 , however we have measured $0.29 \pm 0.03<C^{2}<0.73 \pm 0.09$. We explain this range of values for $C^{2}$ by considering the possible transition rates between the different magnetic sub-levels in the system. This result has significant consequences when measuring trap populations via fluorescence in a MOT.
\end{abstract}

PACS numbers: $32.80 . \mathrm{Xx}$

\section{INTRODUCTION}

The MOT has become a standard experimental tool in atomic physics. For many experiments involving atomic samples produced in a MOT, it is important to have an accurate knowledge of the fraction of atoms in the excited state. A common method for estimating the atom number and density is based on accurately measuring the fluorescence and excited state fraction (ESF) in the MOT [1-7]. In addition, an accurate knowledge of atomic populations is crucial for cold collision experiments where different species can have vastly different collisional parameters [8-11]. The ESF is also an important parameter for studies investigating decay dynamics and lifetimes in MOTs [12].

The fractional population of the excited state in a MOT is related to the scattering rate, $\xi$, for an atom in an optical field and is given by, $\Pi_{e}=\xi / \Gamma$, where, $\Gamma$ is the linewidth of the atomic transition in $\mathrm{rad} \mathrm{s}^{-1}$. In the simple case of a two-level atom in an optical field, the standard textbook definition for the scattering rate is [13],

$$
\xi=\frac{\Gamma}{2}\left[\frac{\Omega^{2} / 2}{\delta^{2}+\Gamma^{2} / 4+\Omega^{2} / 2}\right],
$$

where $\delta$ is the detuning of the incident light field in $\operatorname{rad~s}^{-1}$ and $\Omega$ is the Rabi frequency, given by,

$$
\Omega=\frac{-E_{0}}{\hbar}\langle 2|e \hat{r} \cdot \hat{\epsilon}| 1\rangle
$$

Here $e$ is the elementary charge of an electron, $E_{0}$ is the amplitude of the electric field, $\hat{r}$ is the electron coordinate with respect to the atom's centre of mass, $\hat{\epsilon}$ is a unit vector in the direction of polarization of the electric field and $\langle 1|$ and $|2\rangle$ represent the initial and final states of the single photon dipoleallowed transition. The matrix element $\langle 2|e \hat{r} \cdot \hat{\epsilon}| 1\rangle$ defines the

\footnotetext{
*Electronic address: r.sangegriffith. edu . au
}

coupling between the dipole moment of the atom $\hat{\mu}=e \hat{r}$ and the incident light field.

Equation 1 is commonly used to estimate the ESF given the trapping laser detuning and intensity. In reality, the geometry of a MOT leads to a much more complicated system. The energy of the internal atomic state and the electric field polarization are position dependent and generally the scattering rate is a function of all the possible transitions between the various Zeeman sublevels for the ground and excited states. Various models have been developed to account for the atomic multilevel nature in the MOT [14-16], however the most commonly used was first proposed by Townsend et al [16] in a cesium MOT. In this work the authors account for this complexity by modifying equation 1 for the scattering rate as,

$$
\xi=\frac{\Gamma}{2}\left[\frac{C_{1}^{2} \Omega^{2} / 2}{\delta^{2}+\Gamma^{2} / 4+C_{2}^{2} \Omega^{2} / 2}\right],
$$

where, $C_{1}^{2}$ and $C_{2}^{2}$ are average squared Clebsch-Gordan coefficients. It was expected that $C_{1}^{2}$ and $C_{2}^{2}$ would be of equal magnitude and close to the value of 0.4 found by averaging over all the possible transitions and polarizations in Cs. However, Townsend et al reported that it was insufficient to take the value for $C_{1}^{2}$ and $C_{2}^{2}$ as the average for all transitions and polarizations. Both average squared ClebschGordan coefficients were measured to be approximately equal $C_{1}^{2} \simeq C_{2}^{2}=0.7 \pm 0.2$ and significantly larger than the expected value of 0.4 . The interpretation was that in general, the radiation field optical pumps the atom towards the Zeeman substate that interacts most strongly with the local field resulting in an increased scattering rate.

Equation 3 is commonly used to determine excited state populations in MOTs [5-12, 17]. However, due to the complications involved in accurately measuring a value for the average squared Clebsch-Gordan coefficient it has been common to adopt a value of $C^{2}=0.7 \pm 0.2$ for all atomic species. The validity of using of equation 3 to calculate the ESF was demonstrated by Shah et al [18] in a rubidium MOT using a model independent charge transfer technique. However, the validity of adopting $C^{2}=0.7 \pm 0.2$ as being independent 
of the the parameter space under investigation has not been sufficiently explored.

In addition to an accurate measurement of the excited state population, the measurement of $C^{2}$ allows information regarding the average internal quantum state of atoms in the MOT to be inferred. Since the value of $C^{2}$ primarily depends on the $m_{j}$ state of the atom interacting with the light field, it is possible to investigate MOT population dynamics by adjusting experimental parameters such as the frequency detuning of the trapping laser.

We extend previous measurements by investigating the the value of $C^{2}$ in a metastable neon MOT over a range of laser detunings. It is found that the measured value of $C^{2}$ varies widely over the detuning range investigated indicating significant changes in the population dynamics of the MOT. At large frequency detunings we measure the value of $C^{2}=0.73 \pm 0.09$ which is consistent with the work of Townsend et al [16]. In addition, these results represent the first of their kind using $\mathrm{Ne}$ as the species of interest and a range of detunings are identified where the approximation of a particular value for $C^{2}$ is valid.

\section{TECHNIQUE}

The technique we use to measure the average squared Clebsch-Gordan coefficient is developed on a saturatedfluorescence method established by DePue et al [19]. The method is based on measuring the fluorescence when the cooling transition is saturated where the ESF is $1 / 2$. This is achieved through frequency tuning the MOT laser beams to the atomic resonance and ramping up the intensity, thereby saturating the transition. In this high intensity limit, the scattering rate can be inferred from the resulting fluorescence signal and is simply $\xi=N \Gamma / 2$, where $\mathrm{N}$ is the total atom number. The technique was originally used for accurately measuring the phase space density in a MOT and has since been established as a convenient method for determining atom number [20-22]. The advantage of this method is that it depends on very few assumptions and only the efficiency of the imaging system is required to measure atomic population.

The technique can be extended to measure the excited state population in a MOT [21]. In this scheme, two measurements of the fluorescence are made, the first is made at the desired trapping intensity, I, and detuning, $\delta$, for which the ESF is being determined. The second measurement is a saturated measurement with the detuning set to the atomic resonance and the intensity sufficient for saturation, $I_{\text {sat }}$. The ESF is calculated by the ratio, $\Pi_{e}=f / 2 f_{\text {sat }}$, where $f$ and $f_{\text {sat }}$ are the measured fluorescence at the trapping intensity and saturation intensity, respectively. The value of $C_{1}^{2}$ and $C_{2}^{2}$ can be inferred from equation 3 if the total intensity of the light incident on the trapped atoms is well characterized. To avoid the inaccuracies associated with measuring $\Pi_{e}$ absolutely, we make a number of fluorescence measurements over a large range of intensities and extract the desired parameters by fitting to the resulting data. This method can be used to determine the coefficient $C_{2}^{2}$. A subsequent measurement can be made to determine $C_{1}^{2}$. However, if the common approximation that $C_{1}^{2} \simeq C_{2}^{2}=C^{2}$ is made then only a single measurement is required. By performing the measurement using this fitting method there is no need to make absolute measurements of the atom number or excited state fraction and hence uncertainties as a result of absolute calibrations are removed.

It is expected that $C_{1}^{2}$ and $C_{2}^{2}$ are approximately equal in magnitude and in this work we also make the approximation $C_{1}^{2} \simeq C_{2}^{2}$. There are a number of instances in the literature where an equivalent approximation is made, for example, as part of their investigation Shah et al [18] conclude that a one parameter model is sufficient for modeling the excited state fraction in a MOT. Alternative methods for modeling the excited state fraction $[14,15]$ incorporate a single scaling factor that is attached to the saturation intensity to account for the complexities of the MOT. In [15] the saturation intensity which appears in both the numerator and denominator of the appropriate equation is left as a floating variable during their analysis. This method is equivalent to making the assumption that the two average squared Clebsch-Gordan coefficients in equation 3 are equal. In [14] a more complex threeparameter model is developed where two parameters are effective Clebsch-Gordan coefficients. However, in this model the two Clebsch-Gordan coefficients account for low intensity and high intensity regimes and the third parameter describes the crossover point between the two regimes.

Experimentally it is convenient to measure the intensity and frequency detuning of the MOT laser beams. Therefore $\Omega$ is expressed in terms of the on resonance saturation parameter,

$$
s_{0}=\frac{2|\Omega|^{2}}{\Gamma^{2}}=\frac{I}{I_{0}},
$$

where, $I=1 / 2\left(\epsilon_{0} c E_{0}^{2}\right)$, is the laser intensity and $I_{0}$, is the saturation intensity, given by,

$$
I_{0}=\frac{\pi h c}{3 \lambda^{3} \tau}
$$

Here, $\lambda$ and $\tau=1$ / $\Gamma$ are the wavelength and natural lifetime of the atomic transition, respectively. By substituting equation 4 into equation 3 the scattering rate can be re-expressed and it follows that the ESF is,

$$
\Pi_{e}=\frac{1}{2}\left[\frac{C^{2} s_{0}}{1+C^{2} s_{0}+4 \delta^{2} / \Gamma^{2}}\right] .
$$

Here the approximation has been made that $C_{1}^{2} \simeq C_{2}^{2}=C^{2}$. We measure $C^{2}$ from equation 6 by keeping the detuning, $\delta$, constant and measuring the fluorescence counts proportional to $\Pi_{e}$ as a function of the laser intensity, $0<I<I_{\text {sat }}$.

The apparatus used in this investigation has been described in detail elsewhere $[9,10]$. Essentially it consists of a MOT for trapping metastable neon in the ${ }^{3} \mathrm{P}_{2}$ state that is loaded from a Zeeman slowed atomic beam. Metastable neon has a conveniently located closed optical transition between the ${ }^{3} \mathrm{P}_{2}$ and the ${ }^{3} \mathrm{D}_{3}$ state $(\lambda=640.2 \mathrm{~nm})$ which we use for laser cooling. The light for the slowing beam passes through the trapping region and modifies the trapping potential, however the detuning is typically $8 \Gamma$ and has a negligible effect on the 


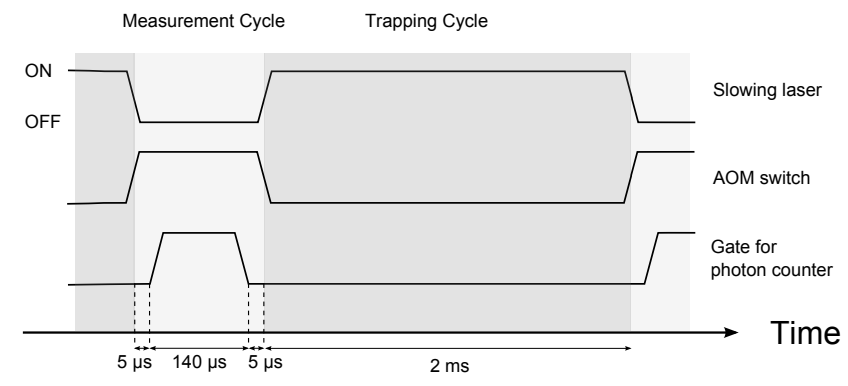

FIG. 1: The timing scheme of the measurement. The measurement cycle lasts a total of $150 \mu$ s with a duty cycle of $500 \mathrm{~Hz}$.

trapped atoms. A Coherent 899 single mode, ring dye laser operated using Kiton red laser dye is used to produce the MOT laser beams. The locking scheme utilizes saturated fluorescence spectroscopy on an isolated sample of metastable neon atoms [23]. The detuning of the laser frequency is controlled by placing a DC magnetic field across the isolated atomic sample which Zeeman shifts the atomic resonance. Typically the total laser intensity (found by summing the contribution from each of the six trapping laser beams) is $I=15 I_{0}$ and the magnetic field gradient across the MOT is operated at $13.5 \mathrm{G} \mathrm{cm}^{-1}$. For these parameters approximately $10^{6}$ atoms are trapped.

To ensure that the number of atoms in the MOT remains constant during the measurement, the laser intensity is modulated on a timescale that does not perturb the atom cloud. The period during which the laser intensity is modulated is referred to as the measurement cycle and lasts $150 \mu \mathrm{s}$. A duration of $150 \mu$ s is chosen based on experimental constraints and is found to be sufficient for acquiring a favorable signal-tonoise ratio while maintaining a regular atom population. During the measurement cycle, the light for the Zeeman slower is switched-off, the MOT light intensity is modulated using an acousto-optic modulator (AOM) (IntraAction model, AOM40) and the fluorescence is measured using an integrated photon counter (Ames Photonics model, Oculus 8010). A $5 \mu \mathrm{s}$ delay before and after activating the integrated photon counter ensures the signal is only due to fluorescence produced during the measurement cycle, see figure 1. For a duty cycle of $500 \mathrm{~Hz}$ we find that the disturbance to the trap is minimal and there are no cumulative effects due to successive measurements that result in a significant change to the number of atoms in the MOT.

To characterize the total intensity of light incident on the trapped atoms, both the intensity profile of the MOT laser beams and the spatial overlap with the atom cloud is required. The MOT laser beams are made relatively large compared to the atom cloud such that the intensity is relatively uniform across the trap. To measure the spatial profile of the atom cloud, the $1 / e^{2}$ diameter is found by observing the fluorescence on a CCD camera and fitting a Gaussian. For $\delta=2 \Gamma$ and $I=15 I_{0}$ the trap $1 / e^{2}$ diameter is measured to be $920 \pm 10 \mu \mathrm{m}$.
The width of the MOT laser beams is found by performing a knife edge measurement. The light for the MOT is transported from the Coherent 899 dye laser to the apparatus via a single mode optical fiber. The subsequent output coupler and lens system results in collimated beam with an approximately Gaussian profile. The $1 / e^{2}$ diameter of the beam is found by fitting to the standard definition of the error function,

$$
P_{x}=\frac{P_{t o t}}{2}\left[1+\operatorname{erf}\left(\frac{x-x_{0}}{(w / 2)}\right)\right],
$$

where $P_{x}$ is the power measured at the position $x, P_{t o t}$ is the total power in the beam, $x_{0}$ is the centre of the beam and $w$ is the $1 / e^{2}$ diameter of the beam. The knife edge measurement is made at a distance of $2.5 \mathrm{~m}$ from the fiber output to simulate the path taken to the MOT region and results in a $1 / e^{2}$ diameter of $8.85 \pm 0.06 \mathrm{~mm}$. The intensity in each of the trapping beams is measured and equalized using a power meter that has been calibrated in accordance with the NIST standard (ISO 100121).

Since the MOT laser beams are relatively large compared to the atom cloud the spatial overlap of each of the trapping beams with the atoms in the MOT must be measured. Special care is taken to ensure that the trap is central by initially aperturing the MOT laser beams and optimizing the system. This ensures that the overlap of the two Gaussians is central and allows the contribution from each beam to be considered equivalent. The incident intensity due to a single laser beam can then be found by integrating over the $1 / e^{2}$ diameter of the atom cloud. Therefore the value of $s_{0}$ is simply found by summing the total effect due to all six trapping beams where a scaling factor is introduced for the retro-reflected beams to account for these beams making multiple passes through the viewports on the trapping chamber.

\section{RESULTS}

To make a measurement of the average squared ClebschGordan coefficient, the measured fluorescence from the integrated photon counter is plotted as a function of the incident light intensity. A background signal is first recorded and subsequently subtracted from the measured fluorescence signal. Figure 2 shows a measurement of $C^{2}$ for a laser detuning of $\delta=1.5 \Gamma$. The measurement of the fluorescence is un-normalized and therefore a two-parameter fit is made using equation 6 . The first fitting parameter corresponds to the value of $C^{2}$ equal to $0.35 \pm 0.04$. The second fitting parameter corresponds to the saturated fluorescence and is related to the ESF. Since we are only interested in the value of $C^{2}$ from the fit, we do not directly measure the ESF and at the point where the fluorescence counts are saturating it cannot be assumed that the ESF is equal to $50 \%$ since the laser is not resonant with the atomic transition.

The error bars in figure 2 are due to counting statistics and are the uncertainty in the fluorescence counts due to processes that are represented by a Poissonian distribution. Laser intensity fluctuations are uncorrelated in time and are included in 


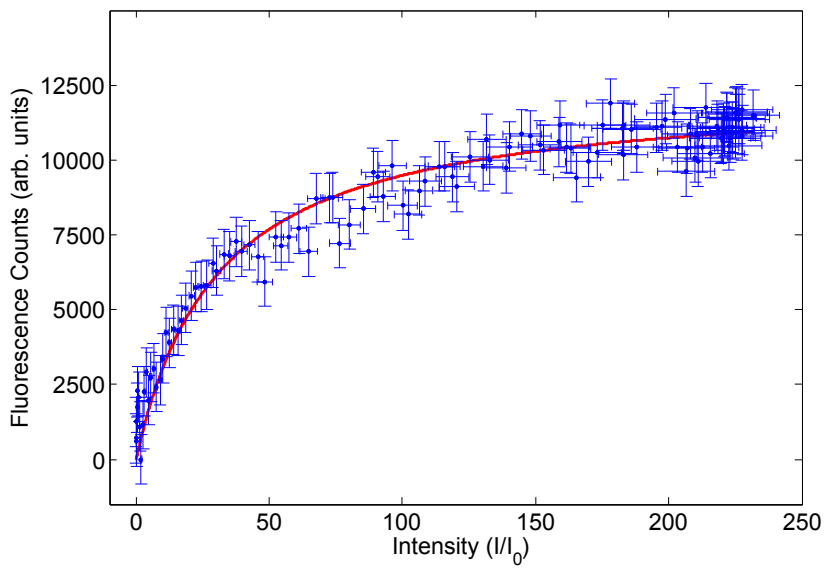

FIG. 2: A graph showing the fluorescence from the Oculus photodiode as a function of the incident light intensity. The detuning is $\delta=1.5 \Gamma$ and the fit using equation 6 gives $C^{2}=0.35 \pm 0.04$. The ESF is not directly measured, instead an additional fitting parameter is introduced to account for the un-normalized fluorescence counts.

these statistics. Systematic uncertainties originate from measurement of the laser beam intensity and detuning. As a result of the Gaussian laser beam, the intensity over the trapping potential is non-uniform. The magnitude of the intensity at the $1 / e^{2}$ radius of the trap is approximately $91.5 \%$ of the intensity in center of the trap. The intensity that we use to calculate the $C^{2}$ coefficient is an averaged intensity and therefore we include a systematic uncertainty of $\pm 5 \%$. This value is overestimated since the majority of the atoms will be located close to the center of the trap where the intensity profile is flatter. The frequency detuning of the laser has a small uncertainty due to the stability of the frequency reference. The long term locking occurs via an error signal derived through the modulation of a saturated absorption spectrum in a gas discharge cell. The uncertainty introduced as a result of this method is estimated to be at most $10 \%$ of the applied detuning. In addition, the MOT quadrupole field shifts the detuning as a function of position in the trap. We estimate the uncertainty at the edge of the trapped atom cloud as a result of the quadrupole field is less than $0.1 \Gamma$. The uncertainty in the measured value of $C^{2}$ is found by taking the statistical uncertainty calculated as a part of the fitting routine to one standard deviation, and adding it in quadrature with the uncertainty in the laser intensity. The uncertainty in the frequency detuning of the laser is included in figure 3.

Because the measured value of $C^{2}$ is dependent on the quantum state of the atom, the population statistics between the available atomic states can be inferred. The variance in $C^{2}$ as the detuning of the MOT laser beams is modified, is an indication of the changing population dynamics of the trapped atoms. Figure 3 shows the variation in $C^{2}$ $\left(0.29 \pm 0.03<C^{2}<0.73 \pm 0.09\right)$ as the laser detuning is scanned between $1 \Gamma<\delta<4 \Gamma$. These results demonstrate the detuning dependence of the light-atom coupling in a MOT and is due to optical pumping between fine structure atomic

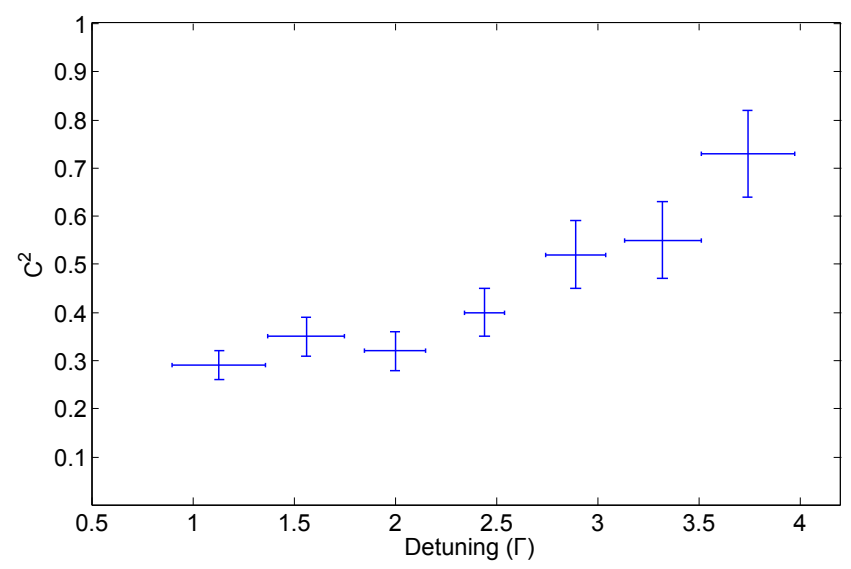

FIG. 3: The average squared Clebsch-Gordan coefficient as a function of the laser detuning.

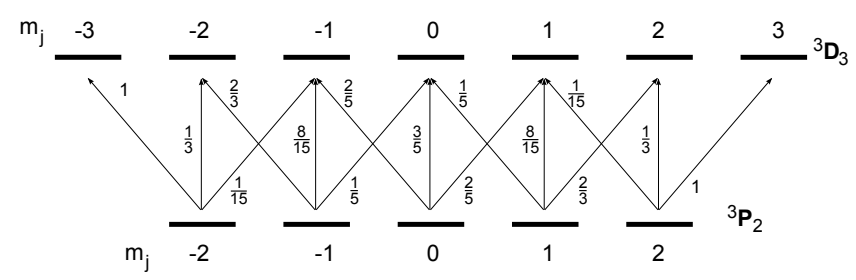

FIG. 4: The squared Clebsch-Gordan coefficient for all the possible $J=2 \rightarrow J=3$ fine structure transitions.

states. A value of $4 \Gamma$ represents an upper limit to the detuning and is dependent on experimental constraints. Below $1 \Gamma$ the trap is observed to become unstable.

The calculated squared Clebsch-Gordan coefficient, $C_{g e}^{2}$, for all the possible $J=2 \rightarrow J=3$ fine structure transitions are shown in figure 4. The subscripts $g$ and $e$ represent the ground and excited $m_{j}$ state and identify the fine structure transition. Since the magnitude of $\Omega$ for any particular transition, depends on the Clebsch-Gordan coefficients, it follows that the strength of the coupling between the atom and the light field is also dependent on the Clebsch-Gordan coefficients. Generally, the atom will be pumped towards the state that couples most strongly with the local field. For $\sigma^{+}$ light the atom is pumped more efficiently into the $m_{j}=2$ to $m_{j}=3$ transition, and for $\sigma^{-}$light the atom is pumped towards the $m_{j}=-2$ to $m_{j}=-3$ transition. At large detunings the atoms couple more strongly with only one laser beam and hence are optically pumped to the larger $m_{j}$ states. Here $C^{2}$ approaches an upper limit and measurements at large detunings are consistent with the previous results where $C^{2}=0.7 \pm 0.2$ [16].

In the case of smaller detunings the measured $C^{2}$ is observed to drop towards a value of $C^{2}=0.29 \pm 0.03$. Here it appears that the atom is pumped by both the incident and retro-reflected beams and tends towards the $m_{j}=0$ state where the calculated squared Clebsch-Gordan coefficient is 
$<1$. For atoms in the $m_{j}=0$ state the description is more complicated as many more transitions are possible. In addition the resonance condition for $\sigma^{+}$and $\sigma^{-}$light varies across the trap and will modify the probability of any specific transition occurring. For example, the probability of driving an atom that is in the $m_{j}=1$ state with a $\sigma^{-}$photon increases. The squared Clebsch-Gordan coefficient for this transition is relatively small, $C_{10}^{2}=0.2$, and as a result, the measured value of $C^{2}=0.29 \pm 0.03$ is lower than the value of 0.46 obtained when averaged over all possible transitions. Therefore the commonly used interpretation that the $C^{2}$ value tends to be larger than the average is insufficient, especially in traps where small detunings are used. Inspection of figure 3 shows a clear regime change that occurs at approximately $2 \Gamma$ where the atom switches from being pumped towards the $m_{j}=0$ state and begins to be more efficiently pumped into states with higher $m_{j}$ numbers.

\section{CONCLUSION}

We have shown that the population dynamics within a MOT changes dramatically with laser detuning and this has significant effects on the measured average squared Clebsch-Gordan coefficients. We explain the range on the $C^{2}$ coefficients us- ing a simple optical pumping model where the strength of the coupling between the atom and light field in the MOT is related to the Rabi frequency, given in equation 2 . The results show that on average the most heavily populated $m_{j}$ state in the MOT is a function of the applied detuning. The technique we have described here can be used as a template for investigating the value of $C^{2}$ in other atomic species over a range of laser detunings.

Our measurements over the detuning range between $1 \Gamma<\delta<4 \Gamma$ in a metastable neon MOT show that the value of $C^{2}$ can vary by approximately a factor of three. This result is significant for measurements of the ESF in a MOT and highlights the importance of making a saturated measurement [19-22] when estimating the ESF. However, sometimes it is convenient to estimate the ESF given only the intensity and detuning of the light incident on the trap, in this case, it is necessary to determine the value $C^{2}$ for the range of experimental parameters under investigation.

\section{ACKNOWLEDGEMENTS}

This research was supported by the Australian Research Council (DP grant \#DP0208713) and Griffith University. JEC was supported by the Australian Research Council.
[1] F. Bardou, O. Emile, J. M. Courty, C. I. Westbrook, and A. Aspect, Europhys. Lett. 20, 681 (1992).

[2] J. J. McClelland and J. L. Hanssen, Phys. Rev. Lett. 96, 143005 (2006).

[3] F. Y. Loo, A. Brusch, S. Sauge, M. Allegrini, E. Arimondo, N. Andersen, and J. Thomsen, J. Opt. B: Quantum Semiclass. Opt. 6, 81 (2004)

[4] X. Xu, T. H. Loftus, J. L. Hall, A. Gallagher, and J. Ye, J. Opt. Soc. Am. B 20, 968 (2003).

[5] A. Browaeys, J. Poupard, A. Robert, S. Nowak, W. Rooijakkers, E. Arimondo, L. Marcassa, D. Boiron, C. Westbrook, and A. Aspect, Eur. Phys. J. D 8, 199 (2000).

[6] Y. Miroshnychenko, A. Gaëtan, C. Evellin, P. Grangier, D. Comparat, P. Pillet, T. Wilk, and A. Browaeys, Phys. Rev. A 82, 013405 (2010).

[7] C. Gabbanini, A. Evangelista, S. Gozzini, A. Lucchesini, A. Fioretti, J. H. Mller, M. Colla, and E. Arimondo, Europhys. Lett. 37, 251 (1997).

[8] S. J. M. Kuppens, J. G. C. Tempelaars, V. P. Mogendorff, B. J. Claessens, H. C. W. Beijerinck, and E. J. D. Vredenbregt, Phys. Rev. A 65, 023410 (2002).

[9] K. J. Matherson, R. D. Glover, D. E. Laban, and R. T. Sang, Rev. Sci. Instr. 78, 073102 (2007).

[10] K. J. Matherson, R. D. Glover, D. E. Laban, and R. T. Sang, Phys. Rev. A 78, 042712 (2008).

[11] R. D. Glover, J. E. Calvert, D. E. Laban, and R. T. Sang, J. Phys. B: At. Mol. Opt. Phys. 44, 245202 (2011).
[12] M. Zinner, P. Spoden, T. Kraemer, G. Birkl, and W. Ertmer, Phys. Rev. A 67, 010501 (2003).

[13] H. J. Metcalf and P. van der Straten, Laser Cooling and Trapping (Springer-Verlag, New York, 1999).

[14] J. Javanainen, J. Opt. Soc. Am. B 10, 572 (1993).

[15] T. P. Dinneen, C. D. Wallace, K.-Y. N. Tan, and P. L. Gould, Opt. Lett. 17, 1706 (1992).

[16] C. G. Townsend, N. H. Edwards, C. Cooper, K. P. Zetie, A. M. Steane, P. Szriftgiser, H. Perrin, C. J. Foot, and J. Dalibard, Phys. Rev. A 52, 1423 (1995).

[17] B. J. Claessens, J. P. Ashmore, R. T. Sang, W. R. MacGillivray, H. C. W. Beijerinck, and E. J. D. Vredenbregt, Phys. Rev. A 73, 012706 (2006).

[18] M. H. Shah, H. A. Camp, M. L. Trachy, G. Veshapidze, M. A. Gearba, and B. D. DePaola, Phys. Rev. A 75, 053418 (2007).

[19] M. T. D. an S. L. Winoto, D. J. Han, and D. S. Weiss, Opt. Commun. 180, 73 (2000).

[20] R. G. Dall and A. G. Truscott, Opt. Commun. 270, 255 (2007).

[21] R. G. Dall, K. G. H. Baldwin, L. J. Byron, and A. G. Truscott, Phys. Rev. Lett. 100, 023001 (2008).

[22] S. S. Hodgman, R. G. Dall, L. J. Byron, K. G. H. Baldwin, S. J. Buckman, and A. G. Truscott, Phys. Rev. Lett. 103, 053002 (2009).

[23] B. T. H. Varcoe, B. V. Hall, G. Johnson, P. M. Johnson, W. R. MacGillivray, and M. C. Standage, Meas. Sci. Technol. 11, N111 (2000). 